\title{
Sodium hydrosulphide against renal ischemia/reperfusion and the possible contribution of nitric oxide in adult male Albino rats
}

\author{
Ibrahim MY, Aziz NM, Kamel MY, Rifaai RA \\ Departments of Physiology, Pharmacology and Histology, Faculty of Medicine, Minia University, \\ Minia, Egypt. emadmax71@yahoo.com
}

\begin{abstract}
OBJECTIVES: This study evaluates the effects produced by $\mathrm{H}_{2} \mathrm{~S}$ donor; sodium hydrosulfide (NaHS), in a renal ischemia/reperfusion (IR) rat model and assesses the possible mediating role of nitric oxide (NO) in these $\mathrm{H}_{2} \mathrm{~S}^{\prime}$ effects. BACKGROUND: For several centuries, hydrogen sulfide $\left(\mathrm{H}_{2} \mathrm{~S}\right)$ had been known to be a highly toxic agent. Recent studies, however, indicated that apart from NO and $\mathrm{CO}, \mathrm{H}_{2} \mathrm{~S}$ is the third "gasotransmitter" involved in the regulation of various physiological functions. Nevertheless, its impact on renal IR injury remains unclear. METHODS: Rats were randomly divided into three groups: sham control; renal IR; and renal IR+NaHS groups. $\mathrm{NaHS}(100 \mu \mathrm{mol} / \mathrm{kg}$, ip) was administered $30 \mathrm{~min}$ prior to the induction of renal ischemia.

RESULTS: NaHS was found to attenuate significantly the IR-induced elevations in the serum levels of urea, creatinine and tumor necrosis factor $\alpha$ (TNF- $\alpha$ ) as compared with IR group. NaHS also significantly compensated the deficits in the total antioxidant capacities (TAC) and lowered the elevated malondialdehyde (MDA) levels observed with renal IR in renal, hepatic, pulmonary, and cardiac tissues. Furthermore, NaHS pretreatment down-regulated the renal IR-induced over-expression of inducible nitric oxide synthase (iNOS) and up-regulated the IR-induced suppression of endothelial nitric oxide synthase (eNOS). The loss of normal architecture, hemorrhage, and inflammatory cells infiltration detected by histopathological examination of renal, hepatic, pulmonary, and cardiac tissues in IR rats were markedly ameliorated by pre-ischemic NaHS treatment.

CONCLUSION: NaHS protects against the effects of renal IR injury by acting primarily through a decrease in both pro-inflammatory cytokines and iNOS expression as well as through up-regulation of the eNOS pathway. Furthermore, $\mathrm{H}_{2} \mathrm{~S}$ has a powerful anti-oxidant and anti-apoptotic effects (Tab. 2, Fig. 6, Ref. 45). Text in PDF www.elis.sk. KEY WORDS:Hydrogen sulfide, ischemia/reperfusion injury, nitric oxide, tumor necrosis factor $\alpha$, malondialdehyde, total antioxidant capacities.
\end{abstract}

\section{Introduction}

Ischemia reperfusion (IR) injury affects $\mathrm{O}_{2}$-dependent cells of tissues and organs such as the heart, brain, liver, kidney, and intestine and it is the major reason for organ dysfunction or even non-function that follows the transplantation(1). Renal IR is a common cause of acute kidney injury (AKI) which occurs in many clinical situations such as transplantation, partial nephrectomy, sepsis, hydronephrosis, or elective urological operations. The mortality during AKI is largely due to extra-renal manifestations (2).

The pathophysiology of IR-induced AKI is highly complex. In addition to the hypoxic hit consequent to ischemia, the reperfusion phase has been associated with additional renal injury. IR-induced activation of inflammatory pathways has been shown to worsen AKI. Furthermore, increased production of radical oxygen species (ROS) and regional imbalance between vasoactive mediators are believed to be of great importance in the development of AKI by

Departments of Physiology, Pharmacology and Histology, Faculty of Medicine, Minia University, Minia, Egypt

Address for correspondence: N.M. Aziz, MD, PhD, Department of Physiology, Faculty of Medicine, Minia University, 61111, Minia, Egypt. Phone: +2.0122256894 , Fax: +2.086 .2324414$ causing impaired microcirculatory perfusion, apoptosis, cellular damage, and irreversible organ failure(3).

Several studies started to investigate mechanisms that underlie distant organ effects of renal IR injury and found a significant inflammatory effect of renal IR on the lung, liver, and heart $(4,5)$. Evidence suggests that the deleterious effects of AKI on remote organ function could, at least in part, be due to the loss of normal balance of immune, inflammatory, and soluble mediator metabolism pathways including nitric oxide that mediates injury of the tubular epithelium (6). Accurate identification of these pathways will be critical in developing the therapeutic approaches in order to improve outcomes in AKI.

Hydrogen sulfide $\left(\mathrm{H}_{2} \mathrm{~S}\right)$ is the third member of the growing family of gaseous signaling molecules that include nitric oxide (NO) and carbon monoxide (CO). Together, these molecules play a major role in regulating numerous vital biological functions in mammals (7). $\mathrm{H}_{2} \mathrm{~S}$ is produced by cystathionine- $\beta$-synthase (CBS), cystathionine- $\gamma$-lyase (CSE) and 3-mercapto-pyruvate-sulfur-transferase (3MST) in mammalian cells $(8) . \mathrm{H}_{2} \mathrm{~S}$ is abundantly produced in the kidney and liver (9). The diverse physiological functions of $\mathrm{H}_{2} \mathrm{~S}$ make it capable of protecting the heart (10), brain (11), liver (12), and lung (13) against IR injury when given at low micromolar or nanomolar (i.e. physiological) doses(14). 
681-688

In the liver, the underlying mechanisms of the protection appear to includesuppression of the oxidative stress via antioxidant activities, reduction of the inflammatory mediators such as tumor necrosis factor- $\alpha$ (TNF- $\alpha$ ), interleukin-10 (IL-10), and reduction ofhepatocyte apoptosis (8).

In the kidney, $\mathrm{H}_{2} \mathrm{~S}$ exerts significant diuretic, natriuretic and kaliuretic effects by raising the glomerular filtration rate and inhibiting the tubular sodium re-absorption (15). Sen et al(16) reported that $\mathrm{H}_{2} \mathrm{~S}$ supplementation has been shown to inhibit the up-regulation of adhesion molecules and inflammatory mediators, and reduce the macrophage infiltration, interstitial fibrosis, and glomerulosclerosis.

Nitric oxide (NO), which is generated by three different isoforms of NO synthase enzymes, namely neuronal (nNOS), inducible (iNOS), and endothelial (eNOS), has been extensively studied for its role in vascular functions and as a signaling molecule. However, the role of this gaseous molecule is being reevaluated with the appreciation of a new gasotransmitter $\mathrm{H}_{2} \mathrm{~S}$ that also serves many important regulatory roles in physiological systems. The interrelation of $\mathrm{NO}-\mathrm{H}_{2} \mathrm{~S}$ and their subsequent biochemical interactions are complex and currently unclear. To date, only a small number of reports suggest that $\mathrm{NO}-\mathrm{H}_{2} \mathrm{~S}$ molecules may influence each other in their production and pathophysiological functions(17). Since there is a lack of information on the effect of $\mathrm{H}_{2} \mathrm{~S}$ on renal IR injury including its distant organ affection, the mechanisms of action of $\mathrm{H}_{2} \mathrm{~S}$ are still open to debates, and as it is still not known whether NO production is involved in these mechanisms, this study was designed to investigate the effects of administration of a water soluble $\mathrm{H}_{2} \mathrm{~S}$ donor; sodium hydrosulfide (NaHS), to a rat model of renal IR.

\section{Material and methods}

\section{Animals}

Four-month-old adult male albino (Sprague Dawley strain) rats, weighing 230-280 g were used throughout the present study. Rats were purchased from the National Research Center, Cairo, Egypt. Rats were housed in stainless steel cages offering individual housing. Each rat had a tag number. They were left freely wandering in their cages for two weeks with normal hours of dark/light cycle for acclimatization before starting the experiment. They were allowed free access to tap water and normal rats' diet (ElNile Company, Egypt). All experimental protocols were approved by the animal care committee of Minia University that coincides with international guidelines.

\section{Chemicals}

Sodium hydrosulfide (NaHS) powder was purchased from Sigma Company, Germany; all other chemicals were of analytical grade and were obtained from commercial sources.

\section{Surgical procedure}

The surgical technique was followed according to the method described by Choket al(18). Following anesthesia induced by intraperitoneal injection of xylazine $(10 \mathrm{mg} / \mathrm{kg} \mathrm{bw})$ and ketamine
(50 mg/kg bw), minimal laparotomy was made using minimal dissection. The anesthetized rat was placed on a heating pad to maintain constant rectal temperature $\left(37^{\circ} \mathrm{C}\right)$ throughout the surgery. Total normothermic renal ischemia was induced for $45 \mathrm{~min}$ by occluding the renal pedicle using a sterile non-traumatizing vascular clamp, and then subjected to reperfusion for $60 \mathrm{~min}$ by removing the clamp.

\section{Experimental groups}

Animals were randomly divided into three groups (per 6 animals each), namely (1) Sham-control group (C); rats were subjected to laparotomy and manipulation of the kidney hilum but without vascular occlusion; (2) renal IR group (IR), (3) renal IR+NaHS group (IR+NaHS); NaHS was administered i.p. at a dose of 100 $\mu \mathrm{mol} / \mathrm{kg} 30 \mathrm{~min}$ before ischemic insult(19). The experiment was terminated by sacrificing the rats by decapitation and collecting blood samples. The kidneys, liver, lung, and heart were excised, weighed, and instantly stored at $-80{ }^{\circ} \mathrm{C}$ for biochemical assay.

\section{Biochemical analyses}

Sera were separated and stored in aliquots at $-80^{\circ} \mathrm{C}$ till used for estimation of serum urea by Berthelot enzymatic colorimetric method, serum creatinine by Jaffé calorimetric-end point meth$\operatorname{od}(20)$, and serum TNF- $\alpha$ by a RIA-IRMA (radioimmunoassayimmuno-radiometric assay) method.

\section{Preparation of tissue homogenates}

Specimens from each organ were weighed and homogenized separately in potassium phosphate buffer, $10 \mathrm{mM}$; $\mathrm{pH}$ (7.4). The ratio of tissue weight to homogenization buffer was $1: 10$. The homogenates were centrifuged at $5,000 \mathrm{rpm}$ for $10 \mathrm{~min}$ at $4^{\circ} \mathrm{C}$. The resulting supernatant was used for determination of malondialdehyde (MDA) according to the method of Ohkawaet al(21) and total antioxidant capacities (TAC) using colorimetric assay kit according to the manufacturer's instructions (Biodiagnostic, Egypt).

\section{Histological examination}

Specimens were fixed in 10\% neutral-buffered formalin, dehydrated, cleared, and embedded in paraffin wax. Tissue sections of 5-6 $4 \mathrm{~m}$ thickness were obtained and deparaffinized. Some sections were stained with hematoxylin and eosin (22).

For immunohistochemical detection of inducible nitric oxide synthase (iNOS) and endothelial nitric oxide synthase (eNOS), we used iNOS, and eNOS monoclonal mouse antibody (Lab Vision Laboratories) according to the manufacturer's protocol. The slides were then counterstained, dehydrated, and mounted (23).

\section{Statistical analysis}

Data were represented as means \pm standard errors of the mean (SEM). Statistical analysis was performed using Graph pad Prism 5 software and significant difference between groups was done by one-way ANOVA followed by Tukey-Kramar post hoc test for multiple comparisons with a value of $\mathrm{p} \leq 0.05$ considered statistically significant. 


\section{Results}

Effect of NaHS pretreatment on serum levels of urea, creatinine, and $T N F-\alpha$ in renal ischemic rats

Renal injury markers (serum urea and creatinine) and serum TNF- $\alpha$ level were significantly higher in IR group than those in sham control group. However, the pretreatment with NaHS significantly decreased the serum urea, creatinine and TNF- $\alpha$ levels as compared with untreated rats exposed to renal IR injury(Tab. 1).

Effect of NaHS pretreatment on oxidative status in renal, hepatic, pulmonary, and cardiac tissues in renal ischemic rats

Renal, hepatic, pulmonary, and cardiac tissue levels of MDA were significantly higher in IR group than those in sham control group, while renal, hepatic, pulmonary and cardiac tissue levels of TAC were significantly lower in IR group than those in sham control rats. On the other hand, NaHS pretreatment significantly

Tab. 1.Effect of NaHS pretreatment on the serum levels of urea, creatinine, and $\mathrm{TNF}-\alpha$ in renal ischemic rats.

\begin{tabular}{lccc}
\hline Groups & $\begin{array}{c}\text { Urea } \\
(\mathrm{mmol} / \mathrm{l})\end{array}$ & $\begin{array}{c}\text { Creatinine } \\
(\mu \mathrm{mol} / \mathrm{l})\end{array}$ & $\begin{array}{c}\text { TNF- } \alpha \\
(\mathrm{pg} / \mathrm{ml})\end{array}$ \\
\hline Control & $31.4 \pm 1.1$ & $0.9 \pm 0.07$ & $12.25 \pm 0.62$ \\
IR & $87.4 \pm 2.5^{\mathrm{a}}$ & $13.2 \pm 0.8^{\mathrm{a}}$ & $41.38 \pm 1.32^{\mathrm{a}}$ \\
IR+NaHS & $58.3 \pm 2.2^{\mathrm{ab}}$ & $3.00 \pm 0.3^{\mathrm{ab}}$ & $19.88 \pm 0.52^{\mathrm{ab}}$ \\
\hline
\end{tabular}

Data are expressed as mean \pm S.E.M. of 6 rats in each group. a) Significant from sham operated control group; b) Significant from renal IR group $(\mathrm{p}<0.05)$, IR - renal Ischemia/reperfusion; NaHS - Sodium hydrosulfide. decreased the renal, hepatic, pulmonary, and cardiac MDA levels and increased the renal, hepatic, lung and cardiac TAC levels as compared with IR group. Nevertheless, there still was a significant difference when compared with the sham control group (Tab. 2).

Effect of NaHS on histological findings in renal, hepatic, pulmonary, and cardiac tissues

Renal tissue of IR group showed marked morphological changes in the renal cortex, degeneration of cells of the proximal and distal tubules, and hemorrhage in between the renal tubules. In addition, some tubular cells became exfoliated and glomerular capillaries were congested. On the other hand, in IR+NaHS-treated group, the renal cortex exhibited mild morphological changes in

Tab. 2. Effect of NaHS pretreatment on the oxidative status in renal, hepatic, pulmonary, and cardiac tissues in renal ischemic rats.

\begin{tabular}{llccc}
\hline Parameters & & Control & IR & IR+NaHS \\
\hline \multirow{3}{*}{ MDA } & Renal MDA & $31 \pm 1.7$ & $96 \pm 3.6^{\mathrm{a}}$ & $53 \pm 2.5^{\mathrm{ab}}$ \\
$(\mathrm{pg} / \mathrm{mg}$ tissue) & Hepatic MDA & $50.1 \pm 2.0$ & $145.7 \pm 7.1^{\mathrm{a}}$ & $87.3 \pm 2.5^{\mathrm{ab}}$ \\
& Pulmonary MDA & $83.1 \pm 3.8$ & $240.2 \pm 6.1^{\mathrm{a}}$ & $170.3 \pm 4.5^{\mathrm{ab}}$ \\
& Cardiac MDA & $49.23 \pm 3.1$ & $99.7 \pm 6.1^{\mathrm{a}}$ & $59.3 \pm 2.5^{\mathrm{ab}}$ \\
\hline \multirow{4}{*}{$\mathrm{TAC}$} & Renal TAC & $82.8 \pm 4.3$ & $42.1 \pm 2.2^{\mathrm{a}}$ & $63.8 \pm 3.0^{\mathrm{ab}}$ \\
$(\mu \mathrm{M} / \mathrm{mg}$ tissue) & Pulmonary TAC & $80.4 \pm 4.1$ & $55 \pm 1.6^{\mathrm{a}}$ & $66.2 \pm 3.3^{\mathrm{ab}}$ \\
& Cardiac TAC & $96.4 \pm 4.6$ & $52 \pm 2.0^{\mathrm{a}}$ & $78.2 \pm 4.5^{\mathrm{ab}}$ \\
\hline
\end{tabular}

Data are expressed as mean \pm S.E.M. of 6 rats in each group. a) Significant from sham-operated control group; b) Significant from renal IR group $(\mathrm{p}<0.05)$.IR - renal Ischemia/reperfusion; MDA -Malondialdehyde; TAC - total antioxidant capacity; NaHS - Sodium hydrosulfide.
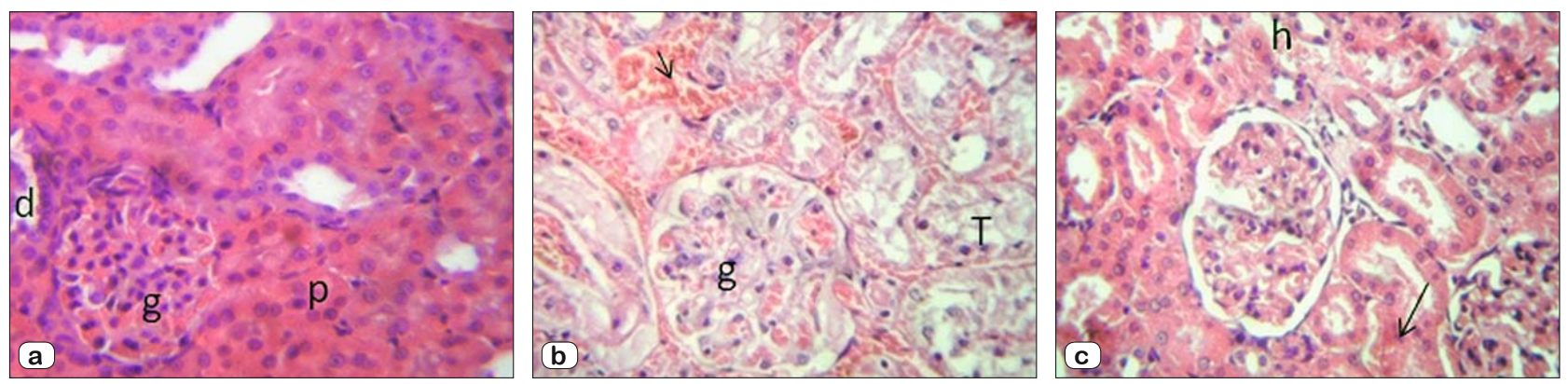

Fig. 1. A photomicrograph of a section in the kidney cortex of a) Control group showing normal structure of renal glomerulus (G) and renal tubules DCT (d)\&PCT (p); b) IR group showing severe renal damage with degeneration of the epithelial cells lining the dilated renal tubules (T), congestion of renal glomerulus (g) and hemorrhage in between the renal tubules (arrow); c) IR+NaHS-treated group showing mild morphological changes in the form of areas of hemorrhage (h) and few degenerated epithelial cells (arrow); H\&E; X400.
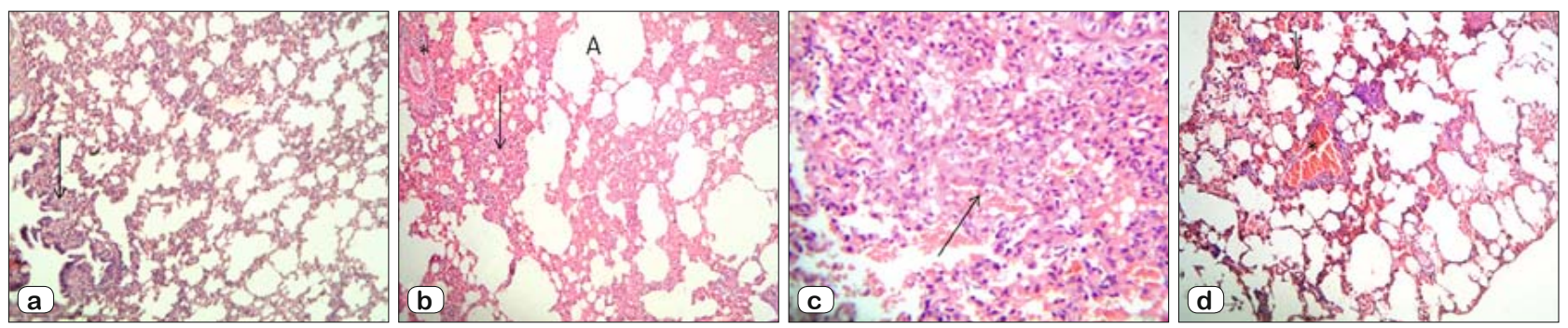

Fig. 2. A photomicrograph of lung sections of a) Control group showing normal alveoli and bronchioles (arrow); X100; b)IR group showing alveolar septal thickening, septal infiltration by erythrocytes and inflammatory cells (arrow), destruction of alveolar septa wall (A), and perivascular inflammatory cells infiltration (*); X100; also IR rat showing alveoli flooded with exudate and red blood cells; X400; c) IR+NaHStreated group showing the lumen of the alveoli retained patent while septal infiltration (arrow), perivascular inflammatory cells infiltration $(*)$ and vascular congestion are greatly improved as compared with that of IR group and most of the alveoli appeared empty; X100. 

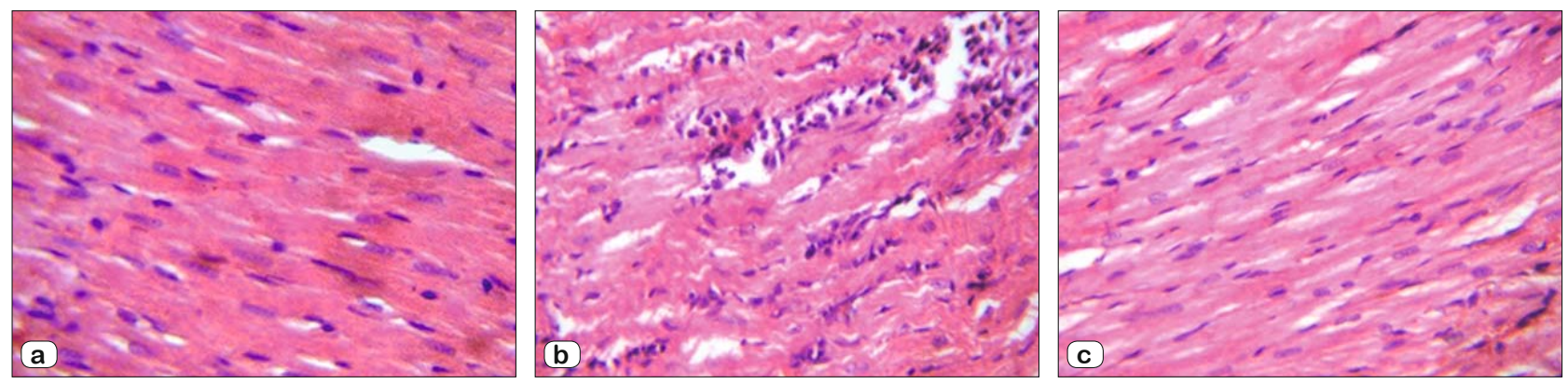

Fig. 3. A photomicrograph of cardiac muscle sections of a)Control group showing branched striated cardiac muscle fibers cut longitudinally with acidophilic sarcoplasm and centrally located pale oval nuclei; b)IR groupwavy widely separated myofibers with inflammatory cellular infiltration (*); c)IR+NaHS-treated group showing myocardial fibers with nearly normal morphology; H\&E; X400.
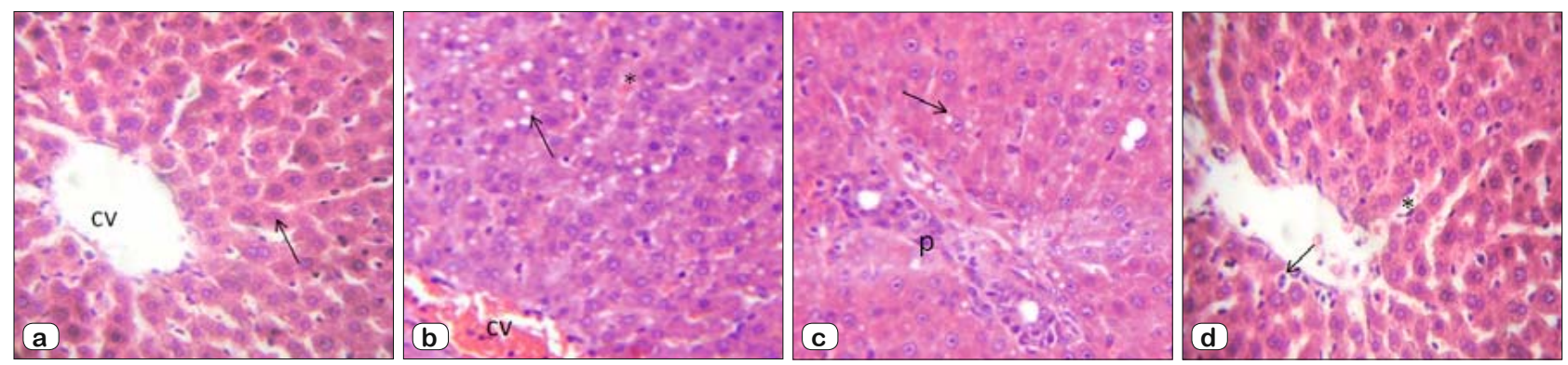

Fig. 4. A photomicrograph of liver sections of; a)Control group showing hepatocytic plates (arrow) radiating from the central vein (CV); b) IR group showing the central vein $(\mathrm{CV})$ and the blood sinusoids $(*)$ are congested. Notice hepatocytes with fatty degeneration (arrow), also IR group showing portal tract with inflammatory cellular infilteration (P). Notice hepatocytes with fatty changes (arrow); c)IR+NaHS-treated group showing mild dilated sinusoids (*), few inflammatory cells infiltration (arrow) No fatty degeneration is observed; Hx\&E; X400.
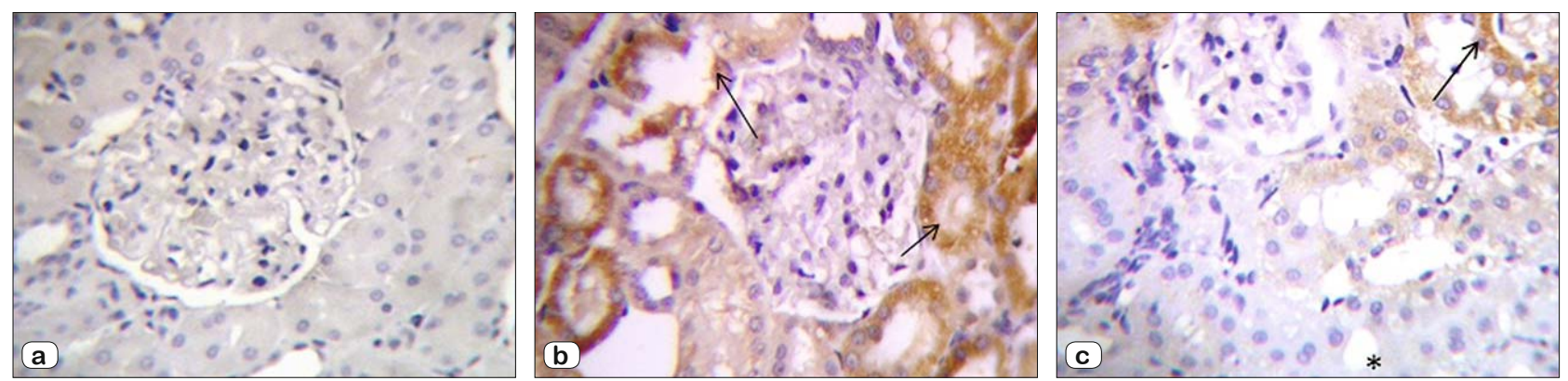

Fig. 5. Localization of iNOSimmunoreactivity in the kidney cortex of; a) Control group showing negative immunoreactivity; b) IR group showing high positive cytoplasmic reaction within epithelial cells of degenerated renal tubules (arrows); c) IR+NaHS-treated group showing positive cytoplasmic reaction in the cells of the degenerated tubules (arrow), while the apparent normal tubules show negative reaction $(*) ; \mathrm{X} 400$.
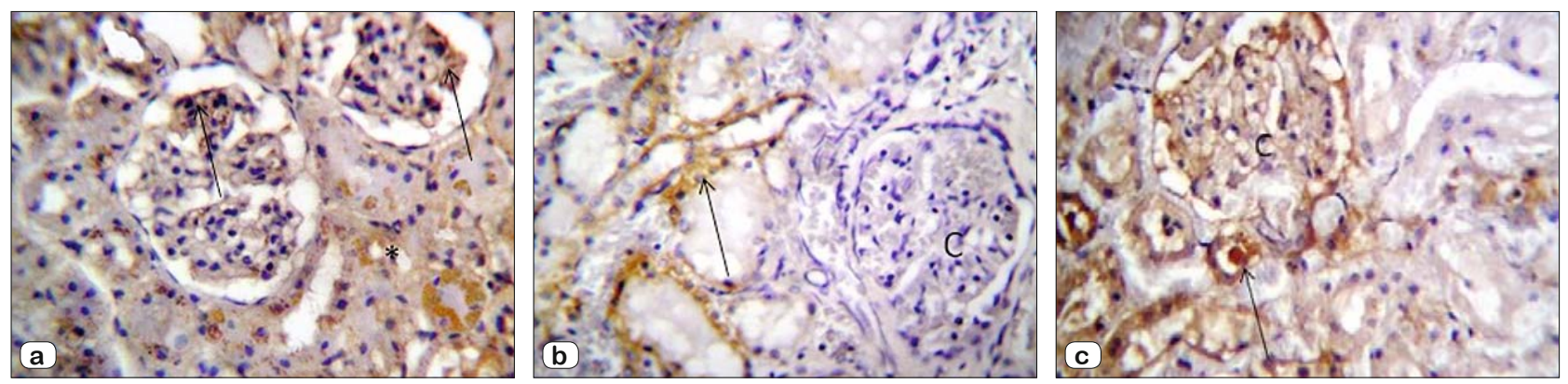

Fig. 6. Localization of eNOSimmunoreactivity in the kidney cortex of; a) Control group showing eNOSimmunoreactivity in the capillary tuft (arrows) and some renal tubules (*); b) IR group showing a positive cytoplasmic reaction within the epithelial cells of some degenerated renal tubules (arrow), with a negative reaction in the capillary tuft (C); c) IR+NaHS-treated group showing positive cytoplasmic reaction in some tubules (arrow) and in the capillary tuft (C); X400. 
form of scattered areas of hemorrhage and some degenerated tubular cells (Fig. 1).

Hepatic lesions in IR group showed congestion of the central vein and blood sinusoids with inflammatory cellular infiltration in the portal tract. The hepatocytes also showed signs of fatty degeneration, while in IR+NaHS-treated group, mild sinusoidal dilatation with few inflammatory cells infiltration was observed. The hepatocytes appeared normal with no fatty degeneration (Fig. 4).

In the lungs, alveolar septa became thickened with septal infiltration by erythrocytes and inflammatory cells. In some areas, the alveolar septa were destructed and the alveolar lumen became flooded with exudate and red blood cells; these changes were evident in IR group. On the other hand, in IR+NaHS-treated group, the lumen of the alveoli retained patent, while septal infiltration, perivascular inflammatory cells infiltration, and vascular congestion were greatly improved as compared with those of IR group (Fig. 2).

In cardiac muscle, myofibers became wavy and widely separated with inflammatory cellular infiltration in IR group. However, in IR+NaHS-treated group, myocardial fibers had nearly normal morphology (Fig. 3).

\section{Effect of NaHS on iNOS and eNOS expression in renal tissues}

Sham control group showed negative iNOSimmunoreactivity, while IR group showed a highly positive cytoplasmic reaction within the epithelial cells of the degenerated renal tubules. However, IR+NaHS group showed negative reaction in apparently normal tubules, while the cells of the degenerated tubules showed a positive cytoplasmic reaction (Fig. 5).

Regarding eNOSimmunoreactivity in the renal cortex, sham control and IR+NaHS groups showed eNOSimmunoreactivity in the capillary tuft and some renal tubules. On the other hand, IR group showed a negative reaction in the capillary tuft and a positive cytoplasmic reaction within the epithelial cells of some degenerated renal tubules (Fig. 6).

\section{Discussion}

Renal IR injury is a common cause of acute renal failure and it contributes considerably to morbidity associated with surgery and anesthesia. Although several decades of research have greatly improved the understanding of the mechanisms underlying the renal IR injury, effective drugs for treatment are still unavailable. Therefore, it is necessary to actively explore other approaches to this problem (24).

In the present study, we report the beneficial effects of NaHS as an $\mathrm{H}_{2} \mathrm{~S}$ donor, prior to IR, in a rat model, because it can dissolve into $\mathrm{Na}^{+}$and $\mathrm{HS}^{-}$in solution. $\mathrm{HS}^{-}$is released and forms $\mathrm{H}_{2} \mathrm{~S}$ with $\mathrm{H}^{+}$. This provides a solution of $\mathrm{H}_{2} \mathrm{~S}$ at a concentration that is about $33 \%$ of the original concentration of $\mathrm{NaHS}(25) . \mathrm{H}_{2} \mathrm{~S}$ is a membrane-permeable gaseous mediator, which has specific cellular and molecular targets. For many years, $\mathrm{H}_{2} \mathrm{~S}$ was considered a toxic agent that causes $85 \%$ mortality at high concentrations. Recently, $\mathrm{H}_{2} \mathrm{~S}$ has been recognized as being the third inorganic gaseous mediator and can thus influence various cellular processes(8).
The deterioration in renal functions following renal IR injury was evidenced in our study and those of others $(26,27)$ by significant elevation in serum urea, creatinine, and renal MDA levels as well as by reduction in the renal TAC level with extensive renal damage shown upon histological examination. This could be explained by many causes. Firstly, by the combination of renal vasoconstriction, tubular obstruction, tubular back-leakage of glomerular filtrate, and reduced glomerular permeability that was observed with renal IR(28), Secondly, IR injury results in activation of multiple cell injury pathways that contribute to organ dysfunction, including those resulting in the production of free radicals that are considered to cause cellular injury by attacking the membranes through peroxidation of polyunsaturated fatty acids(29) during IR injury. This lipid peroxidation results in increased membrane permeability in cells, mitochondria, and lysosomes(30).

Finally, inflammation contributes substantially to the pathogenesis of IR with a central role for particular cells, adhesion molecules, and cytokines. Neutrophils are the inflammatory cells that abundantly produce ROS during IR injury. Renal IR causes tissue injury by oxygen radicals and oxidative stress, caused by an imbalance between the production of ROS and antioxidant capacity(5), ROS produced during renal reperfusion have diverse cytotoxic effects, including DNA damage, protein oxidation, lipid peroxidation, and induction of apoptosis (30).

On the other hand, NaHS administration significantly reversed this deterioration in renal function with improvement in the extent of renal damage observed upon histological examination. These results are in line with Tripataraet al (32) who reported for the first time that the synthesis of endogenous $\mathrm{H}_{2} \mathrm{~S}$ by CSE is essential for the recovery of renal function and integrity following IR, hence the $\mathrm{H}_{2} \mathrm{~S}$-donor NaHS in the present work reduced the renal injury and dysfunction caused by renal IR in rats. This reno-protective effect of $\mathrm{H}_{2} \mathrm{~S}$ could be explained according to Perna and Ingrosso(33) by greater vasodilatation in pre-glomerular arterioles, which increases the renal blood flow and glomerular filtration rate.

Tumor necrosis factor (TNF)- $\alpha$ is a primary cytokine that is produced primarily by activated monocytes/macrophages which play a crucial role in the initiation and continuation of inflammation and immunity. In the kidney, TNF- $\alpha$ is expressed, synthesized, and released by infiltrating macrophages and intrinsic kidney cells, namely, endothelial, mesangial, glomerular, and tubular epithelial cells(34).

TNF- $\alpha$ may up-regulate other cytokines and pro-inflammatory mediators, and thereby cause tissue damage through the gradual rise in serum IL-1 $\beta$ and iNOS levels(35). The authors postulated that iNOS-induced tissue damage might contribute to excessive NO generation and significant production of ROS (36). Higher concentrations of local NO may have no effect on iNOS activity but might actually auto-inhibit eNOS activity, and this came in line with our immune-histological findings that the kidney is rendered more susceptible to actions by vasoconstrictors and the reduced renal blood flow results from increased renal vascular resistance. The decrease in eNOS activity is completely prevented by selective iNOS inhibition in vitro and in vivo(37). 
681-688

A strong link has been established between TNF- $\alpha$ production and oxidative stress during the IR injury process. Thus, inflammatory mediators such as the cytokine TNF- $\alpha$ is thought to have a central role in the pathophysiology of renal injury. TNF- $\alpha$ is consistently up-regulated in response to renal ischemic injury as shown in our study. Activation of TNF- $\alpha$ may induce apoptosis, cell death, as well as inflammation. TNF- $\alpha$ is implicated in the pathogenesis of different renal diseases and can promote renal dysfunction by direct cytotoxicity, vasoconstriction, and inflammatory cells recruitment. Up-regulation of mRNA and protein levels of TNF occurs at a level of whole organ within minutes to hours of the onset of IR injury (38).

The data of the present study clearly demonstrated that NaHS pretreatment significantly reversed this increase in serum TNF- $\alpha$ level as observed in renal ischemic rats. These results are in accordance with Sodhaet al(39) who reported that $\mathrm{H}_{2} \mathrm{~S}$ has been shown to have anti-inflammatory effect by mechanisms which include the limitation of neutrophil adhesion and activation in response to inflammatory stimuli, as well as by suppressing the release of the pro-inflammatory cytokines such as IL-6, IL-8, TNF- $\alpha$. On the other hand, it stimulated the synthesis of the anti-inflammatory chemokine IL-10 (40). In addition,King and Lefer(41) reported that $\mathrm{H}_{2} \mathrm{~S}$ down-regulated the pro-inflammatory cytokines such as iNOS. This came in line with our immune-histological findings as well as in line with the finding that inflammatory cytokines/ chemokines and adhesion molecules react through a mechanism involving nuclear factor $\kappa \mathrm{B}(\mathrm{NF}-\kappa \mathrm{B})$ down-regulation $(42)$.

In the present study and those of others $(27,43,4)$, it is reported that renal IR significantly elevated the MDA level in hepatic, pulmonary and cardiac tissues while the TAC level was significantly decreased when compared to control group, thus suggesting oxidative stress-induced injury of the liver, lung and heart in IR renal injury. Additionally, the inflammatory cell infiltration, degeneration, and congestion shown in the histological examination of distant organs indicate organ damage following IR injury. Here comes the question as to why renal IR led to damage in the distant organs.

Studies had identified a close relationship between renal injury and other organ system failures. Lung functional and structural changes, brain inflammation and functional changes, liver apoptosis, cardiac damage, and inflammation were seen after AKI. Humoral or cellular factors are thought to be the causes of remote organ failure, but their exact pathophysiological mechanisms are not completely understood(2). Grams and Rabb(4) reported that the deterioration in the distant organ> functions occurs during ischemic kidney injury, at least in part, from systemic inflammatory changes that involve cell adhesion molecule and cytokinechemokine expression, activation of the pro-apoptotic pathways, increase in leukocyte infiltration, and oxidative stress.

On the other hand, the data of the present study clearly demonstrated that NaHS significantly decreased MDA and increased TAC levels in liver, lung and heart tissues while improving the histological finding as compared with ischemic rats. Perna and Ingrosso(33)andAminzadeh and Vaziri (9) reported that the $\mathrm{H}_{2} \mathrm{~S}$ donor, NaHS, has been shown to lower ROS production by sup- pressing the expression of ROS-generating enzyme, NADPH oxidase (NOX) and simultaneously by raising antioxidant activities of GSH, catalase and superoxide dismutase. In addition, $\mathrm{H}_{2} \mathrm{~S}$ can scavenge and/or degrade lipid peroxides, up-regulate GSH synthesis and increase nuclear factor E2-related factor-2 (Nrf2) signaling, which confers protection against tissue injury by increasing anti-oxidant and detoxification responses to oxidative stress.

The role of NO in the kidney is complex. It can either attenuate or exacerbate renal injury, depending on the balance between beneficial hemodynamic effects and cytotoxic effects. The site and rate of NO production, its chemical fate, and differences in the temporal expression patterns of NOS determine this balance (37). Both $\mathrm{NO}$ and $\mathrm{H}_{2} \mathrm{~S}$ are chemically reactive gaseous molecules that are generated and distributed across various tissues. The importance of endothelium-derived NO in the regulation of vascular tone and homeostasis has been well documented. NO has been reported to contribute to the maintenance of renal circulation and urine formation. NO was also reported to inhibit stress-induced endothelial cell apoptosis and diminish apoptosis induced by renal IRI (44). Nevertheless, NO can also react with oxygen radicals to form secondary reactive nitrogen species such as $\mathrm{ONOO}^{-}$. Here comes the question as to how $\mathrm{NO}$ participates in $\mathrm{H}_{2} \mathrm{~S}$-mediated reno-protection.

As shown in our study, at therapeutic concentrations (low to mid micromolar), $\mathrm{H}_{2} \mathrm{~S}$ has been reported to decrease iNOS expression while it prevents eNOS degradation and induces eNOS phosphorylation. This leads to subsequent NO production with inhibition of its cytotoxic effects due to peroxynitrite formation, possibly through sulfinyl nitrite formation. This novel product of $\mathrm{H}_{2} \mathrm{~S}$ and $\mathrm{ONOO}^{-}$has the potential to release NO in physiological concentrations sufficient to dilate blood vessels, increase blood flow, and ameliorate IRI, but at the same time it suppresses peroxynitrite formation with its pro-apoptotic and toxic oxidative effects(45). These observations indicate that $\mathrm{H}_{2} \mathrm{~S}$ can mediate its effects through a NO-dependent pathway.

In conclusion, we have shown that a single i.p. NaHS bolus immediately before renal IR (i) limited the IR-induced deterioration in renal functions and (ii) was associated with reduced inflammatory response by acting primarily through a decrease in both pro-inflammatory cytokines and iNOS expression, as well as with up-regulation of the eNOS pathway and (iii) decrease in the oxidative stress response in the renal tissues and distant organs as the liver, heart and lung (iv). It was also associated with improved histological findings in the kidney and distant organs. This discovery suggests that $\mathrm{H}_{2} \mathrm{~S}$ could be an agent useful for preserving the kidney function and protecting distant organs from effects of IR especially during surgical settings such as transplantation or tumor resections requiring vascular clamping.

\section{Limitations}

Despite providing the effects of $\mathrm{H}_{2} \mathrm{~S}$ therapy in renal IR injury, this study has several limitations. Our time course for renal IR is not able to account for long-term effects of sulfide on renal 
function and its distant organ effects. Another limitation of the current study was that the focus on functional data and inflammatory markers did not include a mechanistic investigation into sulfide's method of action in providing renal protection. This will open the door for further future studies, in which multiple dosing groups can be established, and genetically modified animals used.

\section{References}

1. Singh A, Mishra S, Mishra A. Effect of Ginkgo biloba on Renal Ischemia-Reperfusion-induced Oxidant Stress in Rats. Research \& Reviews: J Pharmacol Toxicol Studies 2014; 2 (1): 47-51.

2. Khastar H, Kadkhodaee M, Sadeghipour H, Seifi B, Hadjati J, Delavari $\mathbf{F}$, Soleimani M. Leukocyte involvement in renal reperfusion-induced liver damage. Ren Fail 2011; 33 (1): 79-8z.

3. Almac E, Bezemer R, Kandil A, Aksu U, Milstein D, Bakker J, Demirci-Tansel C, Ince C. Bismaltolatooxovanadium (BMOV) and ischemia/reperfusion-induced acute kidney injury in rats. Intensive Care Med Exp 2014; 2: 3 .

4. Grams M, Rabb $\mathbf{H}$. The distant organ effects of acute kidney injury. Kidney Int 2012; 81 (10): 942-948.

5. Vaghasiya J, Sheth N, Bhalodia Y, Jivani N. Exaggerated liver injury induced by renal ischemia reperfusion in diabetes: effect of exenatide. Saudi J Gastroenterol 2010; 16 (3): 174-180.

6. Mohamed N, Mubarak H. Effects of Renal Ischemia Reperfusion on Brain, Liver \& Kidney Tissues in Adult Male Rats. Life Sci J 2011; 8 (1): 204-212.

7. Aminzadeh M, Vaziri N. Downregulation of the renal and hepatic hydrogen sulfide (H2S)-producing enzymes and capacity in chronic kidney disease. 2012; 27(2): 498-504.

8. Zhang Q, Fu H, Zhang H, Xu F, Zou Z, Liu M, Wang Q et al. Hydrogen sulfide preconditioning protects rat liver against ischemia/reperfusion injury by activating Akt-GSK- $3 \beta$ signaling and inhibiting mitochondrial permeability transition. PLOS One 2013; 8 (9): e74422.

9. Perna A, Ingrosso D. Low hydrogen sulphide and chronic kidney disease: a dangerous liaison. Nephrol. Dial Transplant. 2012; 27 (2): 486-493.

10. Elrod J, Calvert J, Morrison J, Doeller J, Kraus D, Tao L, Jiao X et al. Hydrogen sulfide attenuates myocardial ischemia-reperfusion injury by preservation of mitochondrial function. Proc Natl Acad Sci USA 2007; 104 (39): 15560-15565.

11. Qu K, Chen C, Halliwell B, Moore P, Wong P. Hydrogen sulfide is a mediator of cerebral ischemic damage. Stroke 2006; 37 (3): 889-893.

12. Bos E, Snijder P, Jekel H, Weij M, Leemans J, van Dijk M, Hillebrands $\mathbf{J}$ et al. Beneficial effects of gaseous hydrogen sulfide in hepatic ischemia/reperfusion injury. Transpl Int 2012; 25 (8): 897-908.

13. Fu H, Chen H, Wang C, Xu H, Liu F, Guo M, Wang $Q$ et al. Flurbiprofen, a cyclooxygenase inhibitor, protects mice from hepatic ischemia/ reperfusion injury by inhibiting GSK-3 $\beta$ signaling and mitochondrial permeability transition. Mol Med 2012; 18: 1128-1135.

14. Nicholson C, Calvert J. Hydrogen sulfide and ischemia-reperfusion injury. 2010; 62 (4): 289-297.

15. Betowski J. Hypoxia in the renal medulla: implications for hydrogen sulfide signaling. 2010; 334 (2): 358-363.
16. Sen U, Munjal C, Qipshidze N, Abe O, Gargoum R, Tyagi S. Hydrogen sulfide regulates homocysteine-mediated glomerulosclerosis. 2010; 31 (5): 442-455.

17. Kolluru G, Shen X, Kevil C. A tale of two gases: NO and H2S, foes or friends for life? Redox Biol 2013; 1 (1): 313-318.

18. Chok M, Conti M, Almolki A, Ferlicot S, Loric S, Dürrbach A, Benoît $\mathbf{G}$ et al. Renoprotective potency of amifostine in rat renal ischaemia-reperfusion. Nephrol Dial Transplant 2010; 25 (12): 3845-3851.

19. AbdollahzadeFard A, Ahangarpour A, Gharibnaseri M, Jalali T, RashidiI, Ahmadzadaeh M. Effects of Hydrogen Sulfide on Oxidative Stress, TNF- $\alpha$ Level and Kidney Histological Changes in Cisplatin Nephrotoxicity in Rat. J Phys Pharm Adv 2013; 3 (3): 57-65.

20. Young D. Effects of Disease on Clinical Laboratory Tests. $4^{\text {th }}$ edition. 2001; 3: 67-69.

21. Ohkawa H, Ohishi N, Yagi K. Assay for lipid peroxides in animal tissues by thiobarbituric acid reaction. 1979; 95 (2): 351-358.

22. Bancroft J, Garble M. Theory and Practice of Histological Techniques. 5th edition, Churchill Livingstone: Harcourt, 2007; 85-98: 310314 .

23. Côté A, Silva R, Cuello A. Current protocols for light microscopy immunocytochemistry. In: Cuello AC (Ed). Immunohistochemistry II. Chichester: John Wiley \& Sons, 1993: 147-168.

24. Shokeir A, Hussein A, Awadalla A, Samy A, Abdelaziz A, Khater S, Barakat N. Protection against renal ischaemia/reperfusion injury: A comparative experimental study of the effect of ischaemic preconditioning vs. postconditioning. Arab J Urol 2012; 10 (4): 418-424.

25. Pan L, Xin-Hua Liu X, Gong Q, Wu D, Zhu Y. Hydrogen Sulfide Attenuated Tumor Necrosis Factor-a-Induced Inflammatory Signaling and Dysfunction in Vascular Endothelial Cells. PLoS ONE 6 (5): e19766.

26. Ibrahim I, Elbassuoni E, Ragy M, Habeeb W. Gender difference in the development of cardiac lesions following acute ischemic-reperfusion renal injury in albino rats. 2013; 32 (3): 421-428.

27. Park S, Chen S, Kim M, Brown K, Kolls J, D‘Agati V, Lee $\mathbf{H}$. Cytokines induce small intestine and liver injury after renal ischemia or nephrectomy. Lab Invest 2011; 91 (1): 63-84.

28. Van derHeijden $M$, Versteilen $A$, Sipkema $P$, van NieuwAmerongen G, Musters R, Groeneveld A. Rho-kinase-dependent F-actin rearrangement is involved in the inhibition of PI3-kinase/Akt during ischemia-reperfusion-induced endothelial cell apoptosis. Apoptosis 2008; 13 (3): 404-412.

29. Spek C, Brüggemann L, Borensztajn K. Protease-activated receptor 2 blocking peptide counteracts endotoxin-induced inflammation and coagulation and ameliorates renal fibrin deposition in a rat model of acute renal failure. Shock 2010; 33 (3): 339-340.

30. Yang $\mathbf{S}$, Chou W, Pei L. Effects of propofol on renal ischemia/reperfusion injury in rats. 2013; 6 (5): 1177-1183.

31. Chen G, Fu Y, Wu X. Protective Effect of Salvia miltiorrhiza Extract Against Renal Ischemia-Reperfusion-Induced Injury in Rats. Molecules 2012; 17 (2): 1191-1202.

32. Tripatara P, Patel N, Collino M, Gallicchio M, Kieswich J, Castiglia $\mathbf{S}$, Benetti $\mathbf{E}$ et al. Generation of endogenous hydrogen sulfide by cystathionine gamma-lyase limits renal ischemia/reperfusion injury and dysfunction. Lab Invest 2008; 88 (10): 1038-1048.

33. Perna A. Low hydrogen sulphide and chronic kidney disease: a dangerous liaison. Nephrol Dial Transplant 2012; 27 (2): 486-493. 


\section{1-688}

34. Sanchez-Niño M, Benito-Martin A, Gonçalves S, Sanz A, Ucero A, Izquierdo M, Ramos A et al. TNF superfamily: a growing saga of kidney injury modulators. Mediators Inflamm 2010; 2010. pii: 182958.

35. Fukumoto K, Naito Y, Takagi T, Yamada S, Horie R, Inoue K, Harusato $\mathbf{A}$ et al. Role of tumor necrosis factor- $\alpha$ in the pathogenesis of indomethacin-induced small intestinal injury in mice. 2011; 27 (3): 353-359.

36. Siriussawakul A, Zaky A, Lang J. Role of nitric oxide in hepatic ischemia-reperfusion injury. 2010; 16 (48): 6079-6086.

37. Heemskerk S, Masereeuw R, Russel FG, Pickkers P. Selective iNOS inhibition for the treatment of sepsis-induced acute kidney injury. 2009; 5 (11): 629-640.

38. Bagul A. Ischaemic/reperfusion injury: Role of infliximab. 2012; 2 (3): $35-40$.

39. Sodha N, Clements R, Feng J, Liu Y, Bianchi C, Horvath E, Szabo $\mathbf{C}$ et al. Hydrogen sulfide therapy attenuates the inflammatory response in a porcine model of myocardial ischemia/reperfusion injury. 2009; 138 (4): 977-984.
40. Li L, Fox B, Keeble J, Salto-Tellez M, Winyard P, Wood M, Moore $\mathbf{P}$ et al. The complex effects of the slow-releasing hydrogen sulfide donor GYY4137 in a model of acute joint inflammation and in human cartilage cells. 2013 ; 17 (3): 365-376.

41. King A, Lefer D. Cytoprotective actions of hydrogen sulfide in ischaemia-reperfusion injury. Exp Physiol 2011; 96 (9): 840-846.

42. Baker R, Hayden M, Ghosh S. NF- $\kappa B$, inflammation, and metabolic disease. Cell Metab 2011; 13 (1): 11-22.

43. Azarkish F, Nematbakhsh M, Fazilati M, Talebi A, Pilehvarian A, Pezeshki Z, Moeini M et al. N-acetylcysteine Prevents Kidney and Lung Disturbances in Renal Ischemia/Reperfusion Injury in Rat. 2013; 4 (10): 1139-1146.

44. He K, Chen X, Han C, Xu L, Zhang J, Zhang M, XiaQ. Lipopolysaccharide-induced cross-tolerance against renal ischemia-reperfusion injury is mediated by hypoxia-inducible factor- $2 \alpha$-regulated nitric oxide production. Kidney Int 2014; 85 (2): 276-288.

45. Kolluru G, Shen X, Kevil C. A tale of two gases: $\mathrm{NO}$ and $\mathrm{H}_{2} \mathrm{~S}$, foes or friends for life? Redox Biol 2013; 1 (1): 313-318. 